\title{
Overdiagnosis and overimaging: an ethical issue for radiological protection
}

\author{
Sergio Salerno ${ }^{1}\left[\right.$ Andrea Laghi $^{2}$ (1) $\cdot$ Marie-Claire Cantone ${ }^{3} \cdot$ Paolo Sartori $^{4} \cdot$ Antonio Pinto $^{5} \cdot$ Guy Frija $^{6}$
}

Received: 21 January 2019 / Accepted: 11 March 2019

(c) Italian Society of Medical Radiology 2019

\begin{abstract}
Aims and objectives This study aimed to analyse the key factors that influence the overimaging using X-ray such as selfreferral, defensive medicine and duplicate imaging studies and to emphasize the ethical problem that derives from it.

Materials and methods In this study, we focused on the more frequent sources of overdiagnosis such as the total-body CT, proposed in the form of screening in both public and private sector, the choice of the most sensitive test for each pathology such as pulmonary embolism, ultrasound investigations mostly of the thyroid and of the prostate and MR examinations, especially of the musculoskeletal system.

Results The direct follow of overdiagnosis and overimaging is the increase in the risk of contrast media infusion, radiant damage, and costs in the worldwide healthcare system. The theme of the costs of overdiagnosis is strongly related to inappropriate or poorly appropriate imaging examination.

Conclusions We underline the ethical imperatives of trust and right conduct, because the major ethical problems in radiology emerge in the justification of medical exposures of patients in the practice. A close cooperation and collaboration across all the physicians responsible for patient care in requiring imaging examination is also important, balancing possible ionizing radiation disadvantages and patient benefits in terms of care.
\end{abstract}

Keywords Overimaging $\cdot$ Overdiagnosis $\cdot$ Ethics $\cdot$ Radiation protection

Sergio Salerno

sergio.salerno@unipa.it

1 Department of Diagnostic Radiology, University of Palermo, Policlinico Via del Vespro 127, 90127 Palermo, Italy

2 Department of Surgical and Medical Sciences and Translational Medicine, Sant'Andrea University Hospital, Sapienza-University of Rome, Via di Grottarossa 1035, 00189 Rome, Italy

3 Department of Biomedical, Surgical and Dental Sciences, University of Milan, Via Pascal 36, 20133 Milan, Italy

4 Department of Radiology, SS Giovanni e Paolo Hospital, Castello 6777, 30122 Venice, Italy

5 Department of Radiology, CTO Hospital, Azienda Ospedaliera dei Colli, Naples, Italy

6 Department of Diagnostic Radiology, Hopital Européen Georges Pompidou Paris APHP, Université Paris-Descartes, Paris, France

\section{Introduction}

The field of biomedical imaging, in particular the area of radiology, has expanded intensely over the last decade. The contemporary role of the radiologist is being challenged as the use of images by clinicians is continuously increasing.

The development of medical imaging over the past two decades has produced undisputable benefits to patients in terms of life expectancy and quality of life [1]. This evolution reflects the utilization of complex ionizing and nonionizing radiation technologies such as multidetector computed tomography (MDCT), positron emission tomography (PET), and magnetic resonance imaging (MRI). Part of such growth, however, can be attributed to the overuse of imaging services.

Overuse can be defined as the application of imaging procedures where conditions clearly show that they are unlikely to increase the patient outcome.

Key factors influencing imaging overuse include: the practice behaviour of referring physicians, self-referral (including referral for additional radiological examinations), 
duplicate imaging studies, defensive medicine, missed educational opportunities when inappropriate procedures are requested, patient demand, payment mechanisms, and financial incentives (as in the US healthcare system) [2].

Self-referral was known since 1920 s, well before the advent of high technology in radiology, but observers generally agree its volume has increased in the last $20-30$ years [3]. Self-referral can be manifested in two main ways forms: a physician who is not an imaging specialist (or a non-physician provider) referring patients to his or her own on-site imaging services represents the first form of self-referral; the second form of self-referral consists of physicians referring their patients to outside facilities in which they has personal a financial interest. Self-referral denotes a conflict of interest for the referring physician when the financial profit from conducting the procedure may prevail, the medical necessity of the procedure.

Defensive medicine has been defined as diagnostic or therapeutic actions applied mainly to safeguard against potential claims of malpractice rather than to benefit the patient. Defensive medicine represents a severe and widespread challenge in contemporary medicine, and it is a major cause of overuse of imaging, i.e. its application has a low probability to improve patient outcome. The problems are the concerns of missing unexpected or rare findings and fear from litigation; other reasons are avoidance of an inaccurate diagnosis or keeping costs low. Defensive ordering of diagnostic examinations may additionally lead to overdiagnosis, which is the detection of new findings not associated with a significant impact on health which in turn may determine further unnecessary actions [4].

\section{Discussion}

Imaging services using ionizing radiation (i.e. whole-body CT) are progressively being marketed directly to the public, often with encouragement to individuals to "self-present" to imaging facilities for specific studies [5]. Too often these studies are expensive and provide doubtful benefits to individuals undergoing them. Moreover, physicians should encourage patients to report imaging examinations they have undergone previously in order to avoid duplicate studies. Imaging studies are often duplicated when efforts to identify previous examinations are insufficient or unproductive. Duplicate studies contribute to overuse of medical imaging [6].

Recent research shows that approximately one-third of healthcare spending is duplicative, is unhelpful, or makes patients worse [7]. Unnecessary imaging studies rarely reveal the cause of patients' complaint, yet may disclose incidental findings, which necessitate further imaging or interventional procedures to be clarified [8]. Diagnostic imaging by itself does not improve patient health and provides no intrinsic value to patients. The only possible benefit of imaging is secondary to a change in patient treatment. Therefore, understanding the value of imaging requires incorporating imaging into a framework of medical decision-making and selection of correct therapy. Moreover, diagnostic imaging will always have the potential to lead to false-positive and false-negative diagnoses. Therefore, imaging information must not be considered absolute, but rather must be understood inside a specific clinical context including the patient history, previous radiological examinations, and other clinical data to form a combined picture that reasonably either confirms or excludes a given diagnosis [9].

Because of the possible self-interest by policy makers, payers, physicians, imaging industry, and patients, it clearly emerges the ethical imperatives of trust and right conduct. Overuse of medical imaging increases unsustainable costs in the worldwide healthcare systems and exposes both single individuals and general population to unnecessary radiation doses [10]. It is clear that a serious part of the increasing healthcare costs and fragmentation of medical care resides in cooperation and collaboration across many sectors, including radiologists, industry, referring clinicians, healthcare service payers, and public interest groups.

The bioethical principle of distributive justice holds that healthcare resources should be distributed as equitably as possible. Given finite resources for health services, it is unethical to provide expensive non-essential health services to one sector of the society when another sector lacks of essential services [11]. Despite persistent inequity in the distribution of healthcare resources in the USA, we should ask about new medical interventions, "Does this test or treatment undermine the fair distribution of health resources?" Given the importance of this subject and the magnitude of its potential economic impact, it is important to restore objectivity.

Speaking more in deep about radiological protection, we daily deal not only with useless or inappropriate examinations, but with imaging modalities which may themselves lead to detrimental effects on patients, especially when using contrast media administration and radiation exposure. Indeed, the major ethical problems in radiology emerge in the justification of medical exposures of patients in the practice. The medical exposure of patients is intentional and with the expectation for a direct individual health benefit to the patient. With respect to other planned exposure situations, the justification lies more often with the profession, rather than with government or regulatory authorities, and the final responsibility for the justification, in the use of a particular procedure, lies with the relevant physician, who should be aware of risks and benefits of the involved procedures $[12,13]$. In case of radiological examinations exceeding the needs of good medical practice, the only result may be an 
unjustifiable possible risk for patients, not providing any real benefit, with the result of inconvenience application of the main principles of radiological protection and medical ethics [14]. The potential risks and the uncertainties regarding the risk associated with radiation exposures have a significant role in the decision to undertake a procedure, and also the complexity, in communicating the risk, has to be taken into consideration. In fact, informed consent is more than simply getting a patient to sign a written consent form, it is a communication process, which requires additional time for a real and comprehensive informed consent, and this is also related to the critical question of having the proper time to dedicate to the individual patients. Value judgements on appropriateness of a procedure require knowledge on the implications of the act and about the ethical and societal values on which to base the decisions for that act. The system of radiological protection developed by the International Commission on Radiological Protection (ICRP) has evolved and continues to evolve on the basis of three recognized pillars: science of radiological protection, a set of ethical values, and experience accumulated from the daily practice of radiological protection by professionals $[15,16]$. It is evident that quantifying benefits and harms is often problematic and the threshold between an appropriate and an inappropriate act can vary among patients and patient groups [17]. It is also evident that ethics alone cannot offer a definitive solution to questions and dilemmas, but it can certainly provide useful insights into the principles and philosophy of radiological protection. In such way, it can be of help in the dialogue among experts, and among experts, patients, and people, to highlight values and preferences in view of a positive balance between potential benefits and harms.

Regarding the ethical dimension of radiological protection, it is worthwhile to mention the contribution by Giovanni Silini, who reviewed the ethical foundation of the radiological protection system in his Sievert Lecture, in 1992 [18], by emphasizing that the system has been rationally developed and, at the same time, with the desire to act reasonably. In radiological protection, the core ethical values, as addressed in the recent ICRP Publication on Ethical Foundations of the System of Radiological Protection [15], support the aims of the radiological protection system and its three fundamental principles: justification, optimization, and individual dose limitation. Four ethical values are recognized underpinning the current system of protection: beneficence/non-maleficence, prudence, justice, and dignity. As well known, the origin of medical ethics lies with Hippocratic oath and more recent with the approach by Beauchamp and Childress [19, 20] given through the four principles of biomedical ethics: autonomy, the right of patients to make their own choice; beneficence, to act taking in mind the best interest for the patient; non-maleficence, to consider not to harm; justice, to be fair. The core ethical values recognized in the system of radiological protection are clearly consistent with the principles of biomedical ethics, when considering beneficence and non-maleficence in one single concept; the autonomy replaced by dignity; and the addition of prudence.

Overimaging as applications of imaging procedures where circumstances indicate that they are unlikely to provide additional positive outcomes for the patient may increase the average dose to the population resulting from medical exposures. Given the moral obligation of healthcare providers, "first, do not harm", the ethical indication is to lower the risk, with benefit outweighing the risk, by considering procedures appropriately prescribed and appropriately performed (justification and optimization) [21]. At the same time, there is an ethical relevance related to benefit to the society, which is not achieved and quite disregarded, if an unbalance between health outcome and corresponding costs is created [22-24].

Supply and demand are considered the main mechanisms at the base of overimaging. Expanded availability of technological medical procedures and increased demand from patients/individuals, together with referring clinicians for assurance, can create the feeling and perception of imaging as a mean to comfort patients and clinicians, where the benefits may easily be overestimated, while risks and costs are somewhat neglected [25]. Individual health assessment which is addressed in the last European directive on radiation protection (BSS) could also play a role in increasing unnecessary examinations.

The obligation to benefit the patient must be balanced against the obligation not to cause harm, with the purpose of ensuring that benefits will outweigh, harms (beneficence, non-maleficence) [26] and the attention to these ethical values may become a difficult task if the risks are uncertain, as for the low doses. Overestimation of risks might result in not performing an imaging procedure that could be of benefit and underestimation of risk might result in an increase in risk for patient and society without any advantage for the patient $[27,28]$. Decision-making under uncertainties requires prudence as a central value. Prudence should not be taken as synonymous of conservatism or "never taking risk", but it defines and sustains the way in which decisions are made, and it does not refer only to the outcome of those decisions. We can say that prudence represents the ability to take an informed and well-considered decision under uncertainty, without having a full knowledge of the consequences of the undertaken action. The justification principle combines the ethical values of beneficence and non-maleficence, with the ethical value of prudence. A prudent ethical practice accompanied with proper communication is part of justification and important, where relevant, to avoid possible overimaging [21]. Justice, as core value, requires an equitable treatment for all. A proper imaging examination, at the right time, with attention to justification and optimization, 
can have significant value for the patient and the society, while overutilization of imaging brings inappropriate use of resources that could be used for other medical purposes, thus violating the justice in the distribution of advantages and disadvantages. Justice relates our sense of fairness, and we can say that considering radiation risk a particular attention has to be given to avoid overimaging children in view of their higher risk of adverse effects of radiation, compared with adult [28].

This is particularly true when considering the exponential increase in radiological examinations during the last decades, and the consequent increased number of imaging findings which are not related to the original "diagnostic dilemma". Indeed, market research studies have shown that in the USA the number of computed tomography (CT) examinations increased from 3 million/year in 1980 to 6 million/year in 2006; the actual estimated increased rate is between 8 and 10\%/year [29-31].

It also results from the Medicare program (reimbursing diagnostic imaging services in the USA) that during this period CT scans of the abdomen tripled and those of the thorax quintupled. Furthermore, the number of magnetic resonance imaging (MRI) studies is constantly increasing, with brain examinations rising by fourfold, spinal examinations by sixfold, and knee examinations by tenfold [32]. In the private sector, many studies have now started to offer screening, performed using whole-body CT. A recent study reported that in these tests, in $68 \%$ of the cases, abnormalities causing no symptoms, called incidentalomas, were detected [32, 33]. The most common ones are lung nodules. Small lung nodules are found in $15 \%$ of non-smokers and up to $50 \%$ of smokers [34]. Of course, a finding in a non-symptomatic patient causes negative psychological effects on the patient.

In this regard, for example, it is reported that the risk of death for a liver biopsy performed for the purpose of investigating an incidentaloma (from about 1-2 per 1000 to 4) is of the same order of magnitude as the probability that incidentaloma is a deadly tumour [35].

Also, in the field of CT, an important aspect concerns the detection of anomalies affecting venous circulation and their possible consequences at the pulmonary level. In fact, the prevalence of pulmonary embolism is significantly influenced by the diagnostic tests performed, while from a clinical point of view, few patients with venous thrombosis of the lower limbs have respiratory disorders [36].

Currently in most cases, nuclear medicine was replaced by the spiral CT. This investigation, in patients with thrombosis of the peripheral veins of the lower limbs, identifies $34 \%$ more patients with micro pulmonary embolisms compared to the nuclear medicine test [37]. Therefore, although $\mathrm{CT}$ increases the micropulmonary embolisms detection rate [38], it also entails the risk of contrast media injection, radiation exposure, and increased costs [39].
Ultrasound investigations can also be a source of overdiagnosis. For example, it is reported that about $10 \%$ of individuals without any symptoms can present gallbladder stone on ultrasound examination [40]. A more relevant problem, even for biological and economic costs, is the occasional finding of a thyroid nodule in asymptomatic patients, occurring in $67 \%$ of patients. However, the 10 -year mortality risk of thyroid cancer is only $0.005 \%$. The true probability that incidentaloma is actually a cancer is $<0.01 \%$ [41]. Occasional finding of thyroid nodules almost always leads to the request of at least one subsequent follow-up test, if not directly to the direct execution of a fine needle aspiration. In this way, the economic cost of a thyroid ultrasound study is systematically (at least) doubled. Another of the most commonly discussed topics is the overdiagnosis of prostate cancer. The diffusion of transrectal ultrasound (TRUS) and TRUS-guided biopsy has led to a clear increase in the diagnosis, without, however, significant effects on reduction in mortality rate. The number of identified prostatic neoplasms is directly proportional to the number of samples taken. One study showed that performing six samples in symptomatic patients, no neoplasm was found, but referring the same patients to "saturation biopsy" with 32-38 samples, prostate cancer was detected in 14\% [42].

The American Cancer Society has recently stated that prostate cancer has a slow growth and no tests should be done in men who have a life expectancy of less than 10 years. A recent US study even states that screening programs would increase prostate cancer mortality by $13 \%$, as a result of post-surgical and therapeutic complications in patients who would otherwise have not undergone any treatment [43].

Professor Albin, the discoverer of the PSA, recently published in the New York Times an article entitled "The great error of the prostate" stating that unfortunately PSA test fails to distinguish between two types of prostate cancer: the one that can develop up to be mortal and the other one which remains silent [44]. Ulterior source of overdiagnosis is MR examinations, especially of the musculoskeletal system. Approximately $40 \%$ of people undergoing MRI of the knee, despite being substantially asymptomatic, may have meniscal injuries $[45,46]$. Approximately $50 \%$ of people undergoing MRI of the lumbar spine, although without significant symptoms in the back, may have disc protrusions [47].

The matter of the costs of overdiagnosis is then strongly correlated with that of inappropriateness. In Italy, a sector study has shown a rate of inappropriateness in outpatient radiology requests of $44 \%$ [48].

The Chairman of the Italian Society of Medical and Interventional Radiology (SIRM), in a recent article, confirmed that in the country one-third of the radiological investigations performed is inappropriate [49]. Calculating that in Italy about 100 million radiological examinations per year 
are performed and that the average cost of radiological services, taking into account the various types, is about $80 €$, it is likely to estimate the economic value of the inappropriate tests in about 3 billion EUR/year.

But now, we must consider the most human and important factor of overdiagnosis and overusing of medical radiological examinations: the inappropriate and potentially useless radiation exposure of the population, without any improvement of cost/benefit ratio.

The overuse of medical imaging, when involves methods delivering ionizing radiation, increases the radiation exposure and thus the associated risks such as cancer induction $[50,51]$. The risk of overdiagnosis is particularly important in the setting of cancer screening or emergency [52-54].

This is a critical issue in screening mammography. It refers to detected cancers that would have not progressed to become clinically evident during the patient lifetime [55]. In particular, overdiagnosis is associated with ductal carcinoma in situ, a rare tumour (less than $5 \%$ of the annual incidence of breast cancers) which progresses to invasive disease in a wide time frame ranging between 5 and 15 years according to the grade. The estimation of this phenomenon in this setting is extremely difficult as demonstrated by a wide range of rates from 0 up to $30 \%$. According to European data, it can range between 1 and 10\% [56]. The impact of overdiagnosis on radiation exposure is extremely difficult to estimate. It has been evaluated that the mean glandular dose for screening mammography ranges between 3 and $10 \mathrm{mGy}$ and it is mainly influenced by the number of views acquired, the technology used and by patient-related factors $[57,58]$. Despite the radiation exposure level, it has been demonstrated that the number of deaths caused by radiation-induced cancers is around 125-208 time smaller than the lives saved owing to screening $[58,59]$.

Another setting where this issue is critical is lung cancer screening with low-dose computed tomography (LDCT) since the rate of detected cancers not evolving in clinically relevant disease ranges between 10.99 and $25.83 \%$ [60, 61]. In this setting, the number of false positive is also crucial since it has been demonstrated that $9.2-51 \%$ of positive controls at the first round turns to be a false positive which become $21 \%$ at the second round and $33 \%$ at the third round determining $2.3 \%$ of minor and $2.73 \%$ of major invasive procedures $[63,64]$. More important, in around $50 \%$ of screened patients an incidental finding is detected $7.5 \%$ of which turns to be clinically significant [65]. Overdiagnosis, false positives, and incidental findings increase the number of imaging examination with a consequent radiation exposure increase. The average dose exposure reported in large screening trials for a single low-dose computed tomography ranges between 0.61 and $1.5 \mathrm{mSv}$ and it increases by $4 \mathrm{mSv}$ if a PET-CT is acquired [66]. Thus, the cumulative risk of cancer incidence, attributable to radiation exposure, according to the
BEIR VII report [66], after 10 years of CT screening, is $0.05 \%$; in other words, one radiation-induced lung cancer should be expected every 173 detected cancer and one radiation-induced major cancer every 108 lung cancer detected through screening [67]. Since the effect of screening on lung cancer mortality has been estimated to be about $5 \%$, the risk of radiation-induced cancer can be considered acceptable $[60,62,66]$.

The last major setting where overdiagnosis increases overuse and thus radiation exposure is the field of emergency. In particular, the use of computed tomography has increased fourfold in the last 10 years for any clinical condition such as trauma, pulmonary infections, abdominal pain, and pulmonary embolism $[53,68]$. The indiscriminate use of computed tomography determines an increase in incidental findings leading to a costly and potentially harmful diagnostic, therapeutic, or interventional cascade [68]. The estimation of overdiagnosis in emergency imaging is extremely difficult to evaluate, and there is no evidence in literature. Even more complex is to determine its impact on radiation exposure, which should be considered proportional to the increase in unjustified imaging examinations. In a recent survey, over $85 \%$ of emergency physicians interviewed felt that $22 \%$ of advanced imaging studies ordered were not clinically justified.

\section{Conclusion}

While the radiation exposure risks related to screening imaging seem justified by the benefit of a greater mortality reduction, despite the negative influence of not negligible overdiagnosis, in the setting of emergency imaging this phenomenon as well as overuse negatively influences the radiation exposure of patients without the compensation of clinical benefits.

In practice, searching for reasonableness and tolerability is a permanent effort directed to act wisely, based on accumulated knowledge, ethical values, and experiences. A careful and adequate communication and empathy to the patient, adding a co-involvement of the patient her/himself in the decision-making process, can be of help towards the best choice for the patient's well-being.

\section{Compliance with ethical standards}

Conflict of interest The authors declare that they have no conflict of interest.

Ethical approval This article does not contain any studies with human participants performed by any of the authors but is a review of current clinical cases. 
Ethical standards This article does not contain any studies with animals performed by any of the authors.

\section{References}

1. ESR Executive Council 2009; European Society of Radiology (2010) The professional and organizational future of imaging. Insights Imaging 1:12-20

2. Hendee WR, Becker GJ, Borgstede JP et al (2010) Addressing overutilization in medical imaging. Radiology 257:240-245

3. Kouri BE, Parsons RG, Alpert HR (2002) Physician self-referral for diagnostic imaging: review of the empiric literature. AJR 179:843-850

4. Kainberger F (2017) Defensive medicine and overutilization of imaging-an issue of radiation protection. Wien Klin Wochenschr 129:157-158

5. Lee TH, Brennan TA (2002) Direct-to-consumer marketing of high-technology screening tests. N Engl J Med 346:529-531

6. Sistrom CL, Dreyer KJ, Dang PP et al (2009) Recommendations for additional imaging in radiology reports: multifactorial analysis of 5.9 million examinations. Radiology 253:453-461

7. Fisher ES, Wennberg DE, Stukel T et al (2003) The implications of regional variations in medicare spending. Part 1. The content, quality and accessibility of care. Ann Intern Med 138:273-287

8. Dunnick NR, Applegate KE, Arenson RL (2005) The inappropriate use of imaging studies: a report of the 2004 Intersociety Conference. J Am Coll Radiol 2:401-406

9. Blackmore CC, Castro A (2015) Improving the quality of imaging in the Emergency Department. Acad Emerg Med 22:1385-1392

10. Armao D, Semelka RC, Elias J Jr (2012) Radiology's ethical responsibility for healthcare reform: tempering the overutilization of medical imaging and trimming down a heavyweight. J Magn Reson Imaging 35:512-517

11. Fenton JJ, Deyo RA (2003) Patient self-referral for radiologic screening tests: clinical and ethical concerns. J Am Board Fam Pract 16:494-501

12. Malone $\mathbf{J}$ (2013) Ethical issues in clinical radiology. In: Oughton D, Hansson SO (eds) Social and ethical aspects of radiation risk management. Elsevier Science, Amsterdam, pp 105-130

13. ICRP (2007) Recommendations of the International Commission on Radiological Protection. ICRP Publication 103. Ann ICRP $37: 2-4$

14. Del Rosario PM (2015) Referral criteria and clinical decision support: radiological protection aspects for justification. ICRP 2015. Proceedings of the second international symposium on the system of radiological protection. Ann ICRP 44(1S):276-287

15. ICRP (2018) Ethical foundations of the system of radiological protection. ICRP Publication 138. Ann ICRP 47:(1)

16. Cho KW (2016) Ethical foundations of the radiological protection system. ICRP, 2016. Proceeding of the third international symposium on the system of radiological protection. Ann ICRP 45(1S):297-308

17. Brownlee S, Chalkidou K, Doust J, Elshaug AG, Glasziou P, Halth I, Nagpal S, Saini V, Srivastava D, Chalmers K, Korenstein D (2017) Evidence for overuse of medical services around the world. The Lancet 390(10090):156-168

18. Silini G (1992) Sievert lecture. Ethical issues in radiation protection. Health Phys 63:139-148

19. Beauchamp TL, Childress JF (1979) Principles of biomedical ethics, 1st edn. Oxford University Press, Oxford

20. Beauchamp TL, Childress JF (2012) Principles of biomedical ethics, 7th edn. Oxford University Press, Oxford
21. Dauer LT, Thornton HR, Hay JL, Balter R, Williamson MJ, St Germain J (2011) Fears, feelings, and facts: interactively communicating benefits and risks of medical radiation with patients. AJR 196:756-761

22. Busardò FP, Frati P, Santurro A, Zaami S, Fineschi V (2015) Errors and malpractice lawsuits in radiology: what the radiologist needs to know. Radiol Med 120(9):779-784

23. Olivetti L, Fileni A, De Stefano F, Cazzulani A, Battaglia G, Pescarini L (2008) The legal implications of error in radiology. Radiol Med 113(4):599-608

24. Ramella S, Mandoliti G, Trodella L, D'Angelillo RM (2015) The first survey on defensive medicine in radiation oncology. Radiol Med 120(5):421-429

25. Lysfahl KB (2012) Utilization and utility of diagnostic imaging. Quantitative studies and normative considerations. University of Oslo. ISBN 978-82-8264-065-7

26. Sokol DK (2013) 'First do no harm' revisited. BMJ 25(347):f6426. https://doi.org/10.1136/bmj.f6426

27. Malone J, Zölzer F (2016) Pragmatic ethical basis for radiation protection in diagnostic radiology. Br J Radiol. https://doi. org/10.1259/bjr.20150713

28. Salerno S, Nardi C, Tudisca C, Matranga D et al (2018) Complete written/oral information about dose exposure in CT: is it really useful to guarantee the patients' awareness about radiation risks? Radiol Med 123(10):788-798. https://doi. org/10.1007/s11547-018-0909-0

29. ICRP (2013) Radiological protection in paediatric diagnostic and interventional radiology. ICRP Publication 121. Ann ICRP 42(2): 12

30. Medicare data. https://data.medicare.gov

31. Brenner DJ, Hall EJ (2007) Computed tomography - an increasing source of radiation exposure. NEngl J Med 357:2277-2284

32. Behbahani S et al (2017) "Incidentalomas" on abdominal and pelvic CT in emergency radiology: literature review and current management recommendations. Abdom Radiol 42:1046-1061

33. Furtado CD, Aguirre DA, Sirlin CB et al (2005) Whole body CT screening spectrum of findings and recommendations in 1192 patients. Radiology 237:385-394

34. Hasan N, Kumar R, Kavuru MS (2014) Lung cancer screening beyond low-dose computed tomography: the role of novel biomarkers. Lung 192:639-648

35. Myers RP, Fong A, Shaheen AA (2008) Utilization rates, communications and costs of percutaneous liver biopsy: a population-based study including 4275 biopsies. Liver Int 28:705-712

36. Moser KM, Fedullo PF, LitteJhon JK et al (1994) Frequent asymptomatic pulmonary embolism in patient with deep venous thrombosis. JAMA 271:223-225

37. Anderson DR, Khan SR, Rodger MA et al (2007) Computer tomographic pulmonary angiography vs ventilation-perfusion lung scanning in patients Wind suspected pulmonary embolism: a randomized controller trial. JAMA 298:2743-2753

38. De Monaco NA, D'angelo Q, Kapoor WN et al (2008) Pulmonary embolism incidence in increasing with the use of spiral computer tomography. AJ Med 12:611-617

39. Colagrande S, Origgi D, Zatelli G, Giovagnoni A, Salerno S (2014) CT exposure in adult and paediatric patients: a review of the mechanisms of damage, relative dose and consequent possible risks. Radiol Med 119:803-810. https://doi.org/10.1007/ s11547-014-0393-0

40. Hopper KD, Landis JR, Meilstrup JW et al (1991) The prevalence of asymptomatic gallstones in the general population. Invest Radiol 26:939-945

41. Ezzat S, Sarti DA, Cain DR, Braunstein GD (1994) Thyroid incidentalomas. Prevalence by palpation and ultrasonography. Arch Int Med 154:1838-1840 
42. Fleshner N, Koltz L (2002) Role of "saturation biopsy" in the detection of prostate cancer among difficult diagnostic cases. Urology 60:93-97

43. Andriole GL, Crawford ED, Grubb RL et al (2009) Mortality results from a randomized prostate-cancer screening trial. $\mathrm{N}$ Engl J Med 360:1320-1328

44. Albin RJ (2010) The great prostate mistake. New York Times 10. https://www.nytimes.com/2010/03/10/opinion/10Ablin.html

45. Englund M, Guermazi A, Gale D et al (2008) Incidental Meniscal findings on knee MRI in middle-aged and elderly persons. N Engl J Med 359:1108-1115

46. Kormick J, Trefelner E, McCarthy S et al (1990) Meniscal abnormalities in the asymptomatic population at MR imaging. Radiology 177:463-465

47. Jensen MC, Brant-Zawadski MN, Obuchowski N et al (1994) Magnetic resonance imaging of the lumbar spine in people without back pain. N Engl J Med 1994(331):69-73

48. Cristofaro M, Busi Rizzi E, Schininà V et al (2011) Appropriateness: analysis of outpatient radiology request. Radiol Med. https ://doi.org/10.1007/s11547-011-0725-2

49. Faletti C (2013) L'allarme dei Radiologi. Quotidiano sanità, October 4, 2013

50. Linet MS et al (2012) Cancer risks associated with external radiation from diagnostic imaging procedures. CA Cancer J Clin 62:75-100

51. Vaiserman A et al (2018) Health impacts of low-dose ionizing radiation: current scientific debates and regulatory issues. Dose Response 16:112

52. Ten Haaf K, de Koning HJ (2015) Overdiagnosis in lung cancer screening: why modelling is essential. J Epidemiol Community Health 69:1035-1039

53. Newton EH (2017) Addressing overuse in emergency medicine: evidence of a role for greater patient engagement. Clin Exp Emerg Med 4:189-200

54. Morris E et al (2015) Implications of overdiagnosis: impact on screening mammography practices. Popul Health Manag 18(Suppl 1):S3-S11

55. Carrier M et al (2010) Subsegmental pulmonary embolism diagnosed by computed tomography: incidence and clinical implications. A systematic review and meta-analysis of the management outcome studies. J Thromb Haemost 8:1716-1722

56. Puliti D et al (2012) Overdiagnosis in mammographic screening for breast cancer in Europe: a literature review. J Med Screen 19(Suppl 1):42-56
57. Warren LM, Dance DR, Young KC (2016) Radiation risk of breast screening in England with digital mammography. Br J Radiol 89(1067):20150897

58. Hendrick RE, Helvie MA (2011) United States preventive services task force screening mammography recommendations: science ignored. AJR 196(2):W112-6

59. Miglioretti DL et al (2016) Radiation-induced breast cancer incidence and mortality from digital mammography screening: a modelling study. Ann Intern Med 164:5-14

60. Patz EF Jr et al (2014) Overdiagnosis in low-dose computed tomography screening for lung cancer. JAMA Intern Med 174:269-274

61. De Koning HJ et al (2014) Benefits and harms of computed tomography lung cancer screening strategies: a comparative modelling study for the U.S. Preventive Services Task Force. Ann Intern Med 160:311-320

62. Usman Ali M et al (2016) Screening for lung cancer: a systematic review and meta-analysis. Prev Med 89:301-314

63. Moyer VA (2014) USPST force, screening for lung cancer US preventive services task force recommendation statement. Ann Intern Med 160:330-338

64. Salerno S, Marrale M, Geraci C, Caruso G, Re GL, Casto AL, Midiri M (2016) Cumulative doses analysis in young trauma patients: a single-centre experience. La Radiol Med 121:144-152

65. Humphrey LL et al (2013) Screening for lung cancer with lowdose computed tomography: a systematic review to update the US Preventive services task force recommendation. Ann Intern Med 159:411-420

66. Rampinelli C et al (2017) Exposure to low dose computed tomography for lung cancer screening and risk of cancer: secondary analysis of trial data and risk-benefit analysis. BMJ 356:j347

67. Crowley KD et al (2015) Comments on estimating risks of low radiation doses-a critical review of the BEIR VII report and its use of the linear no-threshold (LNT) hypothesis by Edward J. Calabrese and Michael K. O'Connor. Radiat Res 183(4):476-481

68. Drescher FS, Sirovich BE (2016) Use of computed tomography in emergency departments in the united states: a decade of coughs and colds. JAMA Intern Med 176:273-275

Publisher's Note Springer Nature remains neutral with regard to jurisdictional claims in published maps and institutional affiliations. 